\title{
Bose-Einstein Condensates in Strongly Disordered Traps
}

\author{
T. Nattermann \\ Institut für Theoretische Physik, Universität zu Köln, Zülpicher Str. 77, D-50937 Köln, Germany \\ V.L. Pokrovsky \\ Department of Physics, Texas A6M University, College Station, Texas 77843-4242 and \\ Landau Institute for Theoretical Physics, Chernogolovka, Moscow District, 142432, Russia
}

(Dated: July 6, 2021)

\begin{abstract}
A Bose-Einstein condensate in an external potential consisting of a superposition of a harmonic and a random potential is considered theoretically. From a semi-quantitative analysis we find the size, shape and excitation energy as a function of the disorder strength. For positive scattering length and sufficiently strong disorder the condensate decays into fragments each of the size of the Larkin length $\mathcal{L}$. This state is stable over a large range of particle numbers. The frequency of the breathing mode scales as $1 / \mathcal{L}^{2}$. For negative scattering length a condensate of size $\mathcal{L}$ may exist as a metastable state. These findings are generalized to anisotropic traps.
\end{abstract}

PACS numbers: $03.75 \mathrm{Hh}, 03.75 \mathrm{Kk}$

Bose-Einstein condensation (BEC) is of great interest in a wide variety of systems including superfluidity and superconductivity. Its perhaps cleanest realization is found in dilute ultracold gases of alkali atoms confined in magnetic or optical traps which have been studied in great detail both experimentally [1, 2] and theoretically [3, 4] (and references therein).

More recently these investigations have been extended to traps formed by a superposition of a harmonic and a random potential [5, 6, 7]. Whereas for harmonic traps the behavior of the condensate, e.g. its size, shape, elementary excitations etc. is quite well understood, this is not the case for traps including a finite amount of disorder. Most of the theoretical investigations of BEC in random potentials use as a starting point a description in momentum space (e.g. Bogoliubov transformation or Beliaev-Popov perturbation theory) where the study of the typical disorder effects is notoriously difficult $[8,9,10,11]$. Disorder is there treated perturbatively with $\xi / \mathcal{L}$ as a small parameter, where $\xi$ and $\mathcal{L}$ denote the healing and the Larkin length, respectively. As we will show below in the case of sufficiently strong disorder this parameter is of order one and perturbation theory breaks down (but see [12]).

In the present article we present a semi-quantitative analysis of BEC on the level of Larkin-Imry-Ma arguments 13, 14 to determine the size, shape and the frequencies of the breathing mode in weakly interacting Bose-Einstein condensates in disordered traps. In addition to the oscillator and the scattering length the Larkin length appears as a new relevant length scale which dominates the properties of the condensate for weak interaction and strong disorder. Strong disorder reduces the anisotropy of the condensate. For attractive interaction the condensate may exist in a metastable state.

The model. Starting point is the Hamiltonian of inter- acting bosons

$$
\mathcal{H}=\int d^{3} x \Psi^{\dagger}\left(-\frac{\hbar^{2}}{2 m} \nabla^{2}+U(\mathbf{x})+\frac{2 \pi \hbar^{2} a}{m} \Psi^{\dagger} \Psi\right) \Psi .
$$

Here $\Psi^{\dagger}(\mathbf{x})$ and $\Psi(\mathbf{x})$ are the creation and annihilation operators of the Bose field with $\int d^{3} x|\Psi|^{2}=N$ for the particle number. $m$ denotes the mass of the bosons and $a$ the scattering length. The external potential represents a superposition of the harmonic trap and a Gaussian random potential

$$
U(\mathbf{x})=\frac{m}{2} \sum_{i=1}^{3} \omega_{i}^{2} x_{i}^{2}+U_{\mathrm{dis}}(\mathbf{x}) .
$$

The random potential is characterized by

$$
\left\langle U_{\text {dis }}\right\rangle=0, \quad\left\langle U_{\text {dis }}(\mathbf{x}) U_{\text {dis }}\left(\mathbf{x}^{\prime}\right)\right\rangle=\kappa^{2} \delta\left(\mathbf{x}-\mathbf{x}^{\prime}\right) .
$$

With this ansatz we assume that the correlation length of the disorder is smaller than all other length scales. We will briefly discuss other cases at the end of this article.

In what follows we consider the state of the system at zero temperature. We will first examine the case of an isotropic 3-dimensional trap with $\omega_{1} \approx \omega_{2} \approx \omega_{3}=\omega_{\mathrm{o}}$. Assuming a compact condensate cloud of the radius $R$ the condensate energy per particle $\varepsilon$ can be written as

$$
\varepsilon(R) \approx \frac{\hbar^{2}}{2 m}\left(\frac{1}{R^{2}}+3 \frac{a N}{R^{3}}+\frac{R^{2}}{\ell^{4}}-\frac{4}{3\left(R^{3} \mathcal{L}\right)^{1 / 2}}\right) .
$$

Here we introduced the oscillator length (the size of the harmonic oscillator ground state) $\ell=\left(\hbar /\left(m \omega_{0}\right)\right)^{1 / 2}$. $\mathcal{L}$ denotes the Larkin length $\mathcal{L}=16 \pi \hbar^{4} /\left(27 m^{2} \kappa^{2}\right)$ 13, 14] in $d=3$ dimensions. $N$ is the total particle number. In the following we will set $\hbar=m=1$. In this notation the healing length in the absence of disorder reads $\xi=$ $\sqrt{R^{3} /(6 N a)}$.

The first and the second term in (4) describe the kinetic energy and the interaction of the particles, respectively. 
Both terms favor the spreading of the condensate provided the scattering length is positive. If the radius of the cloud is sufficiently large, namely

$$
R \gg R_{a}=3 a N
$$

the interaction is negligible in comparison with the kinetic energy. Neglecting the inhomogeneity of the condensate, condition (5) can be rewritten as $\xi \gg R$. The third and the fourth term describe the oscillator potential and the typical potential well from the disorder, respectively, both cause its confinement. In the last term we have taken into account that the typical value of the fluctuation of the potential in a region of linear size $R$ scales as $\kappa R^{-3 / 2}$. This term is larger than the kinetic energy only on scales $R \gtrsim \mathcal{L}$. Similarly to the interaction, at sufficiently large radius of the cloud,

$$
R \gg R_{\mathcal{L}}=\ell(\ell / \mathcal{L})^{1 / 7}
$$

the disorder can be neglected in comparison to the harmonic potential.

In general the center of an attractive domain formed by static fluctuations of the random potential may not coincide with the center of the harmonic trap. Nevertheless, our estimates are correct since it is either the harmonic trap or the disorder which leads to the localization of the condensate. Experimentally localization by disorder is often observed by a sudden decrease of the oscillator frequency. In this case the condensate will be localized in the coarse grained potential well closest to the origin.

Condensate size. Below we determine the equilibrium size $R_{0}$ of the condensate as a function of the particle number $N$ and the disorder strength from the equilibrium condition $\partial \varepsilon / \partial R=0$. In the case of weak disorder and relatively small particle number, such that $\ell \ll \mathcal{L}$ and $N \ll \ell /(3 a)$, we can ignore both the disorder and the interaction and hence, from (4) $R_{0} \approx \ell$. At large particle number $N>\ell /(3 a)$, the effect of the interaction sets in and $R_{0}$ approaches the Thomas-Fermi radius [15]

$$
R_{\mathrm{TF}}(N) \sim(a N)^{1 / 5} \ell^{4 / 5}
$$

More interesting is the case of strong disorder, such that the oscillator length is much larger than the Larkin length, $\ell \gg \mathcal{L}$. Then, for small particle number, the competition between the kinetic energy and the effective potential well resulting from the random potential on scale $R$ gives

$$
R_{0} \approx \mathcal{L}, \quad N a \ll \mathcal{L}
$$

Thus the size of the condensate is equal to the Larkin length. We estimate the healing length as $\xi \sim$ $\sqrt{\mathcal{L}^{3} / N a} \gg \mathcal{L}$ from which it is plausible to conclude that the condensate is strongly depleted and the superfluid stiffness vanishes [8].
For larger particle numbers the interaction becomes important and the minimum of (4) is given by

$$
R_{0}(N) \approx\left(\frac{9}{2} N a\right)^{2 / 3} \mathcal{L}^{1 / 3}, \quad \mathcal{L} \ll a N \ll \ell\left(\frac{\ell}{\mathcal{L}}\right)^{5 / 7} .
$$

The (average) healing length is estimated from $\xi \sim$ $\sqrt{R_{0}^{3} / N a} \sim(\mathcal{L} / N a)^{1 / 6} R_{0} \ll R_{0}$ from which we concludes that the condensate is weakly depleted only and the superfluid stiffness remains finite [8]. At the upper boundary for $N$ the radius reaches $R_{\mathcal{L}}$. Finally, for even larger particle number the radius crosses over to the Thomas-Fermi radius. The crudeness of our energy expression (4) does not allow accurate determination of the numerical pre-factors for all quantities derived from (41). If the disorder decreases, the Larkin-length $\mathcal{L}$ increases and the interval where (9) applies diminishes and eventually vanishes when $\mathcal{L}$ reaches the oscillator length $\ell$. In this case only the cross-over at $N a \approx \ell$ discussed previously survives.

Fragmentation. Next we consider the instability of a compact condensate against fragmentation. For $N \ll$ $\mathcal{L} / a$ the ground state energy for increasing $N$ decreases as $E \approx-N /\left(6 \mathcal{L}^{2}\right)$ until it reaches a plateau $E \approx E_{0}$ at $N \gtrsim N_{0} \approx \mathcal{L} / a$ where $E_{0} \approx-2 /(27 \mathcal{L} a)$ (compare (9)). Thus, at $N>N_{0}$, the division of the condensate into $N / N_{0}$ fragments is energetically favorable. The energy of the fragmented condensate is $E \approx\left(N / N_{0}\right) E_{0}\left(N_{0}\right) \approx$ $-N / \mathcal{L}^{2}<E_{0}(N)$. Thus the fragmented condensate corresponds to the true ground state. The total volume of all fragments scales like $\sim N a \mathcal{L}^{2}$ and is hence by a factor $(\mathcal{L} /(N a))$ smaller than the volume of the compact state. The healing length $\xi$ in the fragments is $\xi=\left(\mathcal{L}^{3} /(6 N a)\right)^{1 / 2}$ where $N \lesssim N_{0}$, and hence $\xi \gtrsim \mathcal{L}$. According to [8], in the region where $\xi \gtrsim \mathcal{L}$ a finite fraction of the bosons are not in the condensate. The superfluid stiffness of the fragmented state most likely vanishes. To

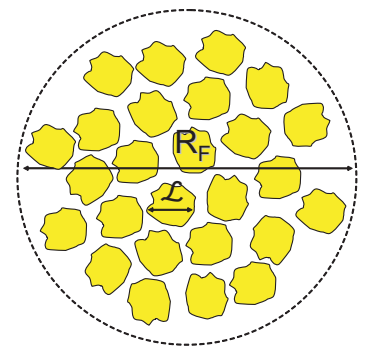

FIG. 1: State of dense fragments. Each fragment includes $\mathcal{L} / a$ particles. The minimal condensate radius is $R_{F} \sim$ $(N a)^{1 / 3} \mathcal{L}^{2 / 3}$.

describe the cross-over to the Thomas-Fermi behavior we consider a state of dense fragments with the total radius $R_{F} \sim(\mathrm{Na})^{1 / 3} \mathcal{L}^{2 / 3} . \quad R_{F}$ denotes the minimal size of of the fragmented state. If there is no confining parabolic potential the fragments may be distributed over a much larger area to take advantage of the tails of the Gaus- 


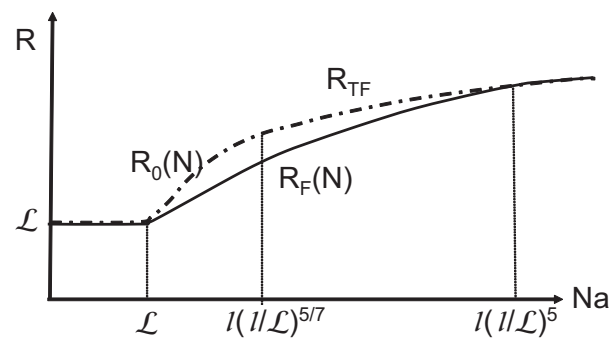

FIG. 2: Condensate radius as a function of the particle number. The bold line corresponds to the equilibrium fragmented state, the dashed line corresponds to the metastable compact state.

sian distribution of disorder. In a fragmented state with radius $R_{F}$ the oscillator energy per particle is of the order $R_{F}^{2} \ell^{-4}$ which has to be compared with the energy per particle $-\mathcal{L}^{-2}$ from the disorder. This gives for the cross-over particle number

$$
N_{c o} a \sim \ell(\ell / \mathcal{L})^{5} .
$$

This number is much larger then the particle cross-over number between (9) and (7) provided $\ell>\mathcal{L}$.

The compact state described by (9) may occur as a metastable state which lives for a short period of time after a confining harmonic trap is switched off (or the oscillator frequency $\omega_{o}$ is strongly reduced). On larger time scales the condensate will lower its energy by decaying into $\sim N a / \mathcal{L}$ fragments each of the size $\mathcal{L}$. This decay into fragments may explain the striped phases found in cigar-like traps [6].

Breathing mode. Next we consider the breathing mode frequency $\omega_{B}$ of the radial oscillations of the condensate around the equilibrium configuration from

$$
m \omega_{B}^{2}=\varepsilon^{\prime \prime}\left(R_{0}\right) .
$$

Without disorder we find from (44) and (11) $\omega_{B}=2 \omega_{\mathrm{o}}$ in the non-interacting and $\omega=\sqrt{5} \omega_{\mathrm{o}}$ in the interacting case, respectively, in agreement with previous results [16].

In the disordered case we obtain for $N a \ll \mathcal{L} \ll \ell$

$$
\omega_{B} \approx \frac{1}{\sqrt{2}}\left(\frac{\ell}{\mathcal{L}}\right)^{2} \omega_{\mathrm{o}} \gg \omega_{\mathrm{o}}
$$

and for $\mathcal{L} \ll a N \ll \ell(\ell / \mathcal{L})^{5 / 7}$ under the assumption of a compact non-fragmented condensate

$$
\omega_{B} \sim \frac{\ell^{2}}{(N a)^{7 / 6} \mathcal{L}^{5 / 6}} \omega_{\mathrm{o}} \gg \omega_{\mathrm{o}} .
$$

Contrary to the pure case the interaction changes the radial oscillations of the condensate strongly.

In the true equilibrium fragmented state the breathing mode will be controlled by the weak interaction between the fragments. In this case the frequency gap will probably decrease as $\sim R_{F}^{-2}$.
Negative scattering length. So far we assumed that the scattering length $a$ is positive. In the case of negative scattering length as e.g. in the case of ${ }^{7} \mathrm{Li}$ atoms [17], there is only a metastable state of finite radius $R_{c}$ following from $\partial \varepsilon /\left.\partial R\right|_{R=R_{c}}=0$. This state becomes unstable at a critical particle number $N_{c}$. The condition for the disappearance of the metastable minimum follows from

$$
\frac{\partial \varepsilon(R)}{\partial R}=\left.\frac{\partial^{2} \varepsilon(R)}{\partial R^{2}}\right|_{R=R_{c}}=0
$$

In the pure case $\mathcal{L} \gg \ell$, condition (14) gives for the critical particle number $N_{c} \approx 0.12 \ell /|a|$ in agreement with [17, 18, 19, 20].

The strong disorder decreases the radius of the metastable droplet as it follows from the equilibrium condition

$$
\left(\frac{\mathcal{L}}{R}\right)^{1 / 2}-\frac{4}{27} \frac{N}{N_{c}}\left(\frac{\mathcal{L}}{R}\right)^{3 / 2}=1
$$

Here $N_{c}$ denotes the critical particle number beyond which the metastable state disappears

$$
N_{c}=\frac{8}{243} \frac{\mathcal{L}}{|a|} \approx 0.033 \frac{\mathcal{L}}{|a|} .
$$

The radius of the metastable droplet in the region $N<$ $N_{c}$ decreases monotonically from $R_{c} \approx \mathcal{L}$ for $N \ll N_{c}$ to $R_{c} \approx \frac{2}{9} \mathcal{L}$ for $N \approx N_{c}$.

Anisotropic traps. So far we assumed that the trap is isotropic whereas experimentally often anisotropic traps are considered. Our results can be easily extended to these cases. For brevity we will consider here only the strongly anisotropic situations (i) $\omega_{1}=\omega_{2}=\omega_{\mathrm{o}} \ll \omega_{3}=$ $\omega_{\perp}(d=2)$ and (ii) $\omega_{1}=\omega_{\mathrm{o}} \ll \omega_{2}=\omega_{3}=\omega_{\perp}(d=1)$ corresponding to a pancake and a cigar-shape like trap, respectively. We assume that the the oscillator length obeys $\ell_{\perp}=\left(\hbar / m \omega_{\perp}\right)^{1 / 2} \ll \ell, \mathcal{L}$ so that the extension of the condensate in the transverse direction is given by $\ell_{\perp}$. The extension $R_{0}$ in the longitudinal direction follows then from the minimum of the energy per particle

$$
\varepsilon \approx \frac{1}{2 R^{2}}+\frac{3 a N}{2 R^{d} \ell_{\perp}^{3-d}}+\frac{R^{2}}{2 \ell^{4}}-\frac{2}{d\left(R^{d} \mathcal{L}_{d}^{4-d}\right)^{1 / 2}}, \quad d=1,2 .
$$

Here we have introduced the $d$-dimensonal Larkin length $\mathcal{L}_{d}=\left(\mathcal{L} \ell_{\perp}^{3-d}\right)^{1 /(4-d)}$ and assumed that the correlation length $b$ of the random potential is small compared with the transverse width of the trap, $b \ll \ell_{\perp}$. In the opposite case $b \gg \ell_{\perp}, \ell_{\perp}$ has to be replaced by $\ell_{\perp}^{2} / b$ in the definition of $\mathcal{L}_{d}$. From (17) we can read off the generalized cross-over radii

$$
R_{a} \sim \ell_{\perp}\left(\frac{\ell_{\perp}}{a N}\right)^{1 /(2-d)}, \quad R_{\mathcal{L}}=\ell\left(\frac{\ell}{\mathcal{L}_{d}}\right)^{\frac{4-d}{4+d}} .
$$

For $R \gg R_{a}$ the interaction and for $R \gg R_{\mathcal{L}}$ the disorder can be neglected, respectively. For a $d$-dimensional trap 
we find in analogy to (8) for small particle numbers

$$
R_{0} \approx \mathcal{L}_{d}, \quad N a<\ell_{\perp}\left(\ell_{\perp} / \mathcal{L}_{d}\right)^{2-d} .
$$

In this region the healing length $\xi \gtrsim \mathcal{L}_{d}$ such that a finite fraction of particles is not in the condensate and superfluidity is suppressed. For larger $N$ (9) has to be replaced by

$$
R_{0} \approx \ell_{\perp}\left(a N / \ell_{\perp}\right)^{2 / d}\left(\mathcal{L}_{d} / \ell_{\perp}\right)^{(4-d) / d} .
$$

This formula is valid as long as

$$
a N<\ell_{\perp}\left(\ell_{\perp} / \ell\right)^{2-d}\left(\ell / \mathcal{L}_{d}\right)^{\frac{(4-d)(d+2)}{4+d}}
$$

For even larger $N$ one find the generalized Thomas-Fermi behavior

$$
R_{T F}(N) \approx \ell_{\perp}\left(a N \ell^{4} / \ell_{\perp}^{5}\right)^{\frac{1}{2+d}}
$$

However, we have to consider here again the instability of the compact domain state described by (20) against fragmentation. The energy of the compact state scales as $-\left(1 / a \ell_{\perp}\right)\left(\ell_{\perp} / \mathcal{L}_{d}\right)^{4-d}$ whereas that of the fragmented state scales as $-N / \mathcal{L}_{d}^{2}$. Thus the fragmented state has the lower energy until the radius of the set of dense fragments

$$
R_{F}(N) \sim \ell_{\perp}\left(N a \mathcal{L}_{d}^{2} / \ell_{\perp}^{3}\right)^{1 / d}
$$

reaches a value such that $\mathcal{L}_{d}^{-2} \sim R_{F}^{2} / \ell^{4}$ which happens at

$$
N_{c o} \sim\left(\ell_{\perp}^{3-d} / \ell^{2-d} a\right)(\ell / \mathcal{L})^{2+d}
$$

which generalizes (10), but is more realistic, especially at $d=1$.

In conclusion, we demonstrated that the disorder strongly influences the size, shape and structure of the condensate cloud. Sufficiently strong disorder leads to the localization of the condensate fragments of linear size of the Larkin length. This result agrees with the conclusions about the suppression of the transport in elongated Bose-condensates derived in [5]. The increasing number of particles enhances interaction and may delocalize the condensate. Such a delocalization transition is especially realistic in a strongly prolonged cigar-shape condensate. If the atoms in condensate attract each other, the disorder decreases the critical number of particles at which collapse is developed.

This work has been supported by DFG-project NA222/5-2 (T.N. and V.P.) and by the DOE under the grant DE-FG02-06ER 46278 (V.P.).

Electronic mail: natter@thp.uni-koeln.de and valery@physics.tamu.edu.

[1] W. Ketterle, Rev. Mod. Phys. 74, 1131 (2002). Nobel lecture: When atoms behave as waves: Bose-Einstein condensation and the atom laser.
[2] E. A. Cornell and C. E. Wieman, Rev. Mod. Phys. 74, 875 (2002), Nobel Lecture: Bose-Einstein condensation in a dilute gas, the first 70 years and some recent experiments.

[3] F. Dalfovo, S. Giorgini L. P. Pitaevskii and S. Stringari, Rev. Mod. Phys. 71, 463 (1999). Theory of Bose-Einstein condensation in trapped gases.

[4] A.J. Leggett, Rev. Mod. Phys. 73307 (2001). BoseEinstein condensation in the alkali gases: Some fundamental concepts.

[5] D. Clement, A. F. Varn, M. Hugbart, J. A. Retter, P. Bouyer, L. Sanchez-Palencia, D. M. Gangardt, G. V. Shlyapnikov, and A. Aspect, Phys. Rev. Lett. 95, 170409 (2005) Suppression of Transport of an Interacting Elongated Bose-Einstein Condensate in a Random Potential.

[6] J. E. Lye, L. Fallani, M. Modugno, D. S. Wiersma, C. Fort, and M. Inguscio, Phys. Rev. Lett. 95, 070401 (2005) Bose-Einstein Condensate in a Random Potential.

[7] C. Fort, L. Fallani, V. Guarrera, J. E. Lye, M. Modugno, D. S. Wiersma, and M. Inguscio, Phys. Rev. Lett. 95, 170410 (2005) Effect of Optical Disorder and Single Defects on the Expansion of a Bose-Einstein Condensate in a One-Dimensional Waveguide.

[8] K. Huang, and H.F. Meng, Phys. Rev. Lett.69, 644 (1992), Hard-sphere Bose gas in random external potentials.

[9] S. Giorgini, L. Pitaevskii, and S. Stringari, Phys. Rev. B 49, 12938 (1994). Effects of disorder in a dilute Bose gas.

[10] A. V. Lopatin and V. M. Vinokur Phys. Rev. Lett. 88, 235503 (2002). Thermodynamics of the Superfluid Dilute Bose Gas with Disorder.

[11] G. M. Falco, A. Pelster, and R. Graham, Phys. Rev. A 75, 063619 (2007), Phys. Rev. A 76, 013624 (2007). Collective oscillations in trapped Bose-Einstein-condensed gases in the presence of weak disorder.

[12] V.I.Yukalov, E.P.Yukalova, K.V.Krutitsky, R.Graham, arXiv:0705.3768 (2007). Bose-Einstein-condensed gases in arbitrarily strong random potentials.

[13] A.I. Larkin, Zh. Eksp. Theor. Fiz 58, 1466 (1970). Effect of inhomogeneities on the structure of the mixed state of superconductors.

[14] Y. Imry and S.K. Ma, Phys. Rev. Lett. 35, 1399 (1975). Random-Field Instability of the Ordered State of Continuous Symmetry.

[15] E. Timmermanns, P. Tommasini and K. Huang, Phys. Rev. A 55, 3645 (1997) Variational Thomas-Fermi Theory of a Nonuniform BEC at Zero Temperature.

[16] S. Stringari, Phys. Rev. Lett. 772360 (1996). Collective Excitations of a Trapped Bose-Condensed Gas.

[17] C. C. Bradley, C. A. Sackett, J. J. Tollett, and R. G. Hulet Phys. Rev. Lett. 75, 1687 (1995). Evidence of Bose-Einstein Condensation in an Atomic Gas with Attractive Interactions.

[18] E.V. Shuryak, Phys. Rev. A54, 3151 (1996). Metastable Bose condensate made of atoms with attractive interaction.

[19] Yu. Kagan, G. V. Shlyapnikov and J. T. M. Walraven, Phys. Rev. Lett. 76, 2670 (1996), Bose-Einstein Condensation in Trapped Atomic Gases.

[20] Yu. Kagan, A. E. Muryshev, and G. V. Shlyapnikov, Phys. Rev. Lett. 81, 933 (1998). Collapse and BoseEinstein Condensation in a Trapped Bose Gas with Negative Scattering Length. 